\title{
Philonsorbonne
}

$7 \mid 2013$

Année 2012-2013

\section{La cathédrale d'ombre. Portée philosophique des interprétations de l'architecture gothique, depuis Goethe et Hugo}

\author{
Julien CHANE-ALUNE
}

\section{(2) OpenEdition \\ Journals}

Édition électronique

URL : https://journals.openedition.org/philonsorbonne/485

DOI : 10.4000/philonsorbonne.485

ISSN : 2270-7336

Éditeur

Publications de la Sorbonne

Édition imprimée

Date de publication : 15 novembre 2013

Pagination : 63-79

ISSN : 1255-183X

Référence électronique

Julien CHANE-ALUNE, «La cathédrale d'ombre. Portée philosophique des interprétations de

l'architecture gothique, depuis Goethe et Hugo », Philonsorbonne [En ligne], 7 | 2013, mis en ligne le 18 décembre 2013, consulté le 08 juin 2021. URL : http://journals.openedition.org/philonsorbonne/485 ; DOI : https://doi.org/10.4000/philonsorbonne.485 


\title{
La cathédrale d'ombre \\ La portée philosophique des interprétations de l'architecture gothique, depuis Goethe et Hugo
}

\author{
Julien Chane-Alune
}

Un présupposé tenace, qui a tout l'air d'être de bon sens, eu égard à l'obscurité parfois impénétrable de certaines cathédrales gothiques, laisse croire que celles-ci, avec leurs murs ouverts par d'immenses et nombreux vitraux et leurs volumes d'une hauteur vertigineuse, sont pensées et bâties pour constituer des totalités lumineuses où chaque élément concoure à l'unité de l'ensemble. Une certaine répugnance moderne semble interdire de voir dans l'obscurité régnant à l'intérieur des cathédrales, effaçant les contours, confondant les formes, et laissant la multiplicité des détails primer sur l'unité de l'ensemble autre chose que les effets néfastes du temps et du mauvais entretien.

Est-ce vraiment le cas? Bien sûr, le manque d'informations et de sources directes sur les ambitions des bâtisseurs de cathédrales rend très difficile toute réponse trop tranchée. Pourtant, les discours sur une métaphysique de la lumière qui aurait présidé aux édifications gothiques sont, depuis une dizaine d'années au moins, de plus en plus discutés. Les recherches historiques mettent désormais l'accent sur le rôle esthétique, théologique et dramaturgique d'une pénombre omniprésente qui n'aurait rien d'accidentel. Selon ces recherches, tout porterait à croire que les cathédrales ont été pensées de manière à être des monuments d'obscurité. S'il y a bien une utilisation ingénieuse et originale de la lumière, par exemple à travers le vitrail, cette lumière n'a pas pour but d'illuminer le volume de la cathédrale afin d'en manifester une hypothétique unité : elle veut au contraire rendre éclatant le vitrail seul, par contraste avec la pénombre environnante, et mettre en valeur certaines parties, comme le chœur, en les détachant sur fond d'obscurité. Les progrès de l'histoire de l'art sur ce point méritent amplement d'être analysés du point de vue philosophique, car ils impliquent au moins deux questions: d'où vient cette idée qu'une cathédrale devrait 
être baignée d'une lumière diaphane ? Et surtout : quelle valeur esthétique et noétique accorder à l'obscurité gothique et à l'absence d'harmonie qu'elle implique?

À la fin du XVIII ${ }^{\mathrm{e}}$ siècle le jeune Goethe va donner le la d'une lecture " classiciste » de l'art gothique qui, malgré que son auteur l'ait reniée dès son retour d'Italie, se répandra notamment chez ceux qui considèreront le gothique comme un style original allemand. Mais en France, un autre auteur non moins influent dans son siècle donne de la cathédrale gothique une lecture tout à fait différente, et en fait un personnage conceptuel d'une dimension spectaculaire inégalée: Notre-Dame de Paris, écrit en 1831, impose à la pensée un monstre de ténèbres. Depuis Hugo, d'autres historiens et philosophes de l'art ont pris au sérieux cette part d'ombre et peignent de la cathédrale gothique un tout autre portrait. Si la pensée veut voir dans la cathédrale un monument de diaphanéité céleste, n'est-ce pas, peut-être, parce qu'elle craint la valeur noétique de l'ombre?

\section{Le grand écart interprétatif de Goethe et des classiques allemands : la cathédrale entre totalité harmonieuse et multiplicité irréductible}

En gardant l'appellation «gothique» (au lieu de «tudesque» par exemple), la postérité ne pouvait pas faire de meilleur compliment aux cathédrales du Moyen-Âge, car en conservant un terme condamnant un style obscur, barbare, sans ordre et sans harmonie ${ }^{1}$, elle a reconnu leur ambition de rompre l'harmonie classique en abandonnant l'exigence des justes proportions. La règle de la cathédrale gothique est bien celle, assumée, de la disproportion, à commencer dans sa verticalité, bien trop importante par rapport à la largeur de la base de l'édifice. Les ingénieuses trouvailles des contreforts et des arcs-boutants servent précisément à rendre possible cette verticalité excessive, qui choque la vue:

Quand on entre dans une cathédrale gothique, la première sensation que l'on éprouve est vraiment celle d'une hauteur qui coupe la respiration, effet qui est dû à la dimension verticale de la nef centrale (Notre-Dame de Paris a 35 mètres de hauteur, la cathédrale de Reims 38, Notre-Dame d'Amiens 42) ainsi qu'au rapport entre la largeur et la hauteur de la nef elle-même ; en effet la première est toujours extrêmement réduite par rapport à la seconde : dans la cathédrale de Chartres, le rapport est de 1 à 2,6, dans Notre-Dame de Paris de 1 à 2,75 , et dans la cathédrale de Cologne, il arrive à 1 sur $3,8^{2}$.

1. «Il y a tant de saillies, de ruptures, de modillons, de vrilles, que tout semble disproportionné. [...] Que Dieu préserve tout pays de cette conception et de cette manière de bâtir! Leur difformité à l'égard de la beauté de nos monuments fait que ces ouvrages ne méritent pas qu'on en parle plus longtemps ", écrit Vasari dans Les vies des meilleurs peintres, sculpteurs et architectes (trad. A. Chastel, Paris, Berger-Levrault, 1985, vol. I, p. 101). 2. M. C. Gozzoli, « L’Art gothique », in Connaître l'art, Paris, Comptoir du livre, 1998, p. 93. 
Cette disproportion volontaire entre hauteur et largeur a pour effet de mettre le fidèle, non pas dans une disposition de confortable contemplation, mais de fascination inquiète. Le dégoût de l'historien de la Renaissance ou de l'esthète classique devant l'œuvre gothique est le signe suffisant de la réussite de cette rupture avec les règles classiques de proportion et d'harmonie. Pourtant, loin d'éprouver cette répugnance, le jeune Goethe commence par louer, dans son texte fameux « Sur l'architecture allemande », la majesté de la cathédrale de Strasbourg. Cette apologie repose sur trois arguments principaux. Premièrement, la dimension personnelle de l'œuvre : à travers la cathédrale, c'est son architecte, Erwin von Steinbach, qui laisse un témoignage de sa pensée «totale, grande, et d'une beauté nécessaire jusque dans sa partie la plus infime $»^{3}$ - notons que Goethe parle bien ici de la pensée de l'architecte et non pas de l'œuvre elle-même. Deuxièmement, l'effort de création originale, modèle d'expression « d'une âme allemande, puissante et rugueuse », par opposition à la fois à "la scène cléricale, bornée et pleine de ténèbres du Moyen-Âge $»^{5}$, et aux contemporains, artistes allemands, vils compilateurs qui «collectionnent des plantes étrangères pour leur propre perte $»^{6}$. Troisièmement, l'unité majestueuse et harmonieuse de l'œuvre elle-même, «créée comme totalité caractéristique par l'unité du sentiment ${ }^{7}$ de son auteur. Pourtant, ces éléments s'avèrent être faux tous les trois. On le sait désormais, loin du style allemand loué par le jeune Stürmer und Dränger (initiant par là une interminable querelle franco-allemande sur sa nationalité d'origine), la cathédrale de Strasbourg s'apparente au style français, et Erwin von Steinbach, mort en 1318, ne fut que l'un des nombreux maîtres d'œuvre qui se sont succédés, de 1176 à 1439 , sur ce chantier de deux siècles et demi ${ }^{8}$.

Mais c'est le troisième argument, d'ordre esthétique, qui s'avère le plus intéressant. Goethe croit si bien voir dans l'édifice l'expression d'une volonté d'harmonie d'un tout constitué de parties s'unissant les unes avec les autres, que le terme même de "gothique », au-delà des disputes historiques et nationales', lui semble une injustice insupportable. Loin d'une œuvre monstrueuse faite de disproportions et d'excès, elle dégage au contraire une volonté d'unité harmonieuse car «tout ici, jusqu'au plus infime filament,

3. Goethe, "Architecture allemande», in Écrits sur l'art, textes choisis et traduits par Jean-Marie Schaeffer, Paris, Klincksieck, 1983, p. 65. Nous nous réfèrerons à cette petite anthologie, rééditée depuis chez Flammarion, pour nos références à Goethe.

4. Ibid., p. 72 .

5. Ibid.

6. Ibid.

7. Ibid., p. 71.

8. Cf. R. Recht, Les Bâtisseurs des cathédrales gothiques, Éditions Les musées de la ville de Strasbourg, Strasbourg, 1989.

9. «Et comment pourrais-je ne pas me fâcher, saint Erwin, lorsque les esthéticiens allemands diminuent ton œuvre en lui accolant le nom de gothique, se fiant aux calomnies de voisins envieux?». - Goethe, « Architecture allemande», in Écrits sur l'art, op. cit., p. 70. 
était forme et concourait à la finalité du Tout $»^{10}$. Mais c'est parce que Goethe se met en condition de n'y voir que ce qu'il veut, qu'il peut parler tout à son aise d'une totalité harmonieusement réglée. En restant sur le parvis et en la contemplant de l'extérieur sans jamais y entrer, il considère la cathédrale comme une sorte d'immense sculpture, et non comme un bâtiment fonctionnel. Eût-il pénétré à l'intérieur, cette totalité qu'il avait à cœur d'embrasser à travers l'élancement de l'ensemble aurait sans doute complètement disparu à ses yeux. Et il n'aurait pas pu continuer d'ignorer sa vocation cultuelle, passée sous silence dans le texte de 1772.

En réalité, tout se passe comme si Goethe s'émerveillait de la beauté classique des cathédrales gothiques, beauté dont il ne découvrira la règle que lors de son voyage en Italie. Les critiques visant un art soi-disant " indéterminé, désordonné, contre-nature, compilé, raccommodé " ${ }^{11}$, qu'il dénonçait tantôt, il les reprendra à son compte quand il s'agira de combattre dans le camp des classiques. On peut relire à cet égard l'introduction qu'il publia en 1798 pour la revue Les Propylées, dénonçant le style néogothique des jeunes artistes, style qui lui apparaît désormais désordonné et sans goût : "En rehaussant de plus en plus les bas-reliefs, en en détachant ensuite différentes parties, puis des figures, en y ajoutant finalement des bâtiments et des paysages, de sorte que la représentation était à moitié de la peinture et à moitié un jeu de marionnettes, on s'est éloigné de plus en plus de l'art véritable $»^{12}$.

Faute de règle, la totalité qu'il voyait à Strasbourg a désormais disparu au profit d'une addition de parties sans unité. Sans l'harmonie qui faisait que chaque élément particulier paraissait prendre naturellement sa place dans l'ensemble totalisant de l'édifice, ceux-ci apparaissent maintenant dans leur singularité, et l'impression de totalité a complètement disparu. Pour un classique, la clarté et l'accessibilité d'un plan d'ensemble doit se laisser voir et se laisser identifier comme la projection de la pensée dans l'édifice, laquelle s'y reconnaît et s'y retrouve comme en elle-même. Il s'agit là de l'idée classique selon laquelle «toute œuvre d'art éminente représente la nature humaine $»^{13}$ et que les œuvres d'art réussies «donnent à l'homme un sentiment d'aise vis-à-vis de lui-même ${ }^{14}$. Autrement dit, l'œuvre d'art doit parler un langage humain, situer l'homme au sein d'une cosmologie, et tout cela afin de lui rendre le monde plus lisible, plus compréhensible, plus domestique.

Or la dynamique pour ainsi dire surhumaine de la cathédrale semble bien contrevenir à cette règle. Le Goethe héraut du classicisme, contrairement au jeune étudiant strasbourgeois, en avait parfaitement saisi la

10. Ibid.

11. Ibid., p. 81 .

12. Ibid., «Introduction aux Propylées », p. 156.

13. Ibid., « Sur Laocoon », p. 165.

14. Ibid., «Introduction aux Propylées », p. 160. 
cause : la fonction religieuse de la cathédrale. Une de ses maximes, à la même époque, dit d'ailleurs que « les temples de l'Antiquité font converger la divinité dans l'homme; les églises du Moyen-Âge en revanche tendent vers le Dieu des cieux ${ }^{15}$. Mais, dans le même temps qu'il reconnaît l'expression de la transcendance divine, il condamne son effet sur la pensée. Surenchérissant à un article de son ami Meyer dans les Propylées, il demande: "Qui a jamais senti son esprit envahi d'une sérénité calme et active dans un édifice barbare, dans les couloirs obscurs d'une église gothique ou d'un château médiéval ? ${ }^{16}$.

Tout l'enjeu du style gothique, comprend Goethe, est d'égarer le regard (et, à travers lui, la pensée) afin de le priver des repères qui lui sont garantis par une règle de proportions anthropométriques. L'évocation de la transcendance divine se fait en perdant la perception dans une verticalité, un mouvement et un foisonnement de détail tels qu'ils interdisent tout repos et toute appréhension globale. Le mouvement et le parcellaire priment. Lorsque le plan d'ensemble ou l'idée générale ne sont pas immédiatement perceptibles, et que la forme générale n'est pas mise en valeur par la lumière mais se fond au contraire dans la pénombre et l'excès de détails, le spectateur se trouve réduit à considérer tous les éléments indépendamment les uns des autres, à passer constamment de l'un à l'autre en quête d'un lien formel, et cette impossibilité d'une vue synthétique constitue en soi un obstacle pour la pensée qui ne trouve pas dans la perception de quoi contenter son besoin de totalité. Robson-Scott a pu montrer la radicalité du revirement de Goethe et sa dénonciation du parcellaire après son voyage en Italie :

La vision du gothique qu'il défendait alors était l'exact opposé de celle de l'auteur de l'essai sur Erwin, pour qui la cathédrale tenait du sublime et de la noblesse de l'expression d'un « esprit titanesque ». C'était, par-dessus tout, un organisme vivant, un tout organique. Désormais le gothique est multiplizierte Kleinheit [petitesse multipliée], sans relations entre les parties, et l'ornement gothique n'est plus ni fonctionnel ni organique, mais simplement «incrusté » comme un enjolivement superficiel ${ }^{17}$.

Malgré ce revirement de Goethe (temporisé en 1823 par une notice expliquant qu'il faut bien accorder une certaine valeur historique à

15. Ibid., « Maximes et réflexions», p. 326.

16. Cité par W. D. Robson-Scott, The literary Background of the Gothic Revival in Germany, op. cit., p. 167. Nous traduisons de l'anglais.

17. W. D. Robson-Scott, The literary Background of the Gothic Revival in Germany, op. cit., p. 165-166. Nous traduisons de l'anglais: Indeed the view of Gothic which then now holds is the precise opposite of that of the author of the Erwin essay, for whom the minster was something sublime and noble in the expression of a 'titan spirit'. Above all it was a living organism, an organic whole. Now Gothic is 'multiplizierte Kleinheit', without relationship between the parts, and Gothic ornament is neither functional nor organic, but merely 'stuck on' as a superficial embellishment. 
l'architecture gothique ${ }^{18}$ ), les romantiques allemands après lui intègreront l'idée que la cathédrale inscrit dans la pierre, par le biais de l'expression esthétique, le besoin métaphysique d'harmonie d'un monde à la mesure de l'homme dont le volume diaphane rendrait les contours et la circonférence appréhensibles. Pourtant le terme de "gothique» va se maintenir. Plus tard, Hegel affirmera à son tour l'origine allemande de ce style mais préfèrera s'en tenir à l' $l^{\prime}$ ancienne appellation qui est plus courante ${ }^{19}$. Paradoxalement, l'idée d'une totalité unifiée de la cathédrale perdurera elle aussi : Schlegel écrit dès 1806 à propos de la cathédrale de Cologne qu' « il faut considérer les statues en pierre des églises gothiques comme de simples structures et ornements. La partie doit servir le tout $\gg^{20}$.

\section{Les tentatives de « récupération classique » de la cathédrale gothique contredites par les avancées de l'histoire de l'art}

On se rend compte de la pénétration de cette lecture «classique »du gothique quand on lit, par exemple au détour d'un texte de Schopenhauer, que selon lui « la philosophie de Kant rappelle d'une manière très frappante l'architecture gothique »: on y reconnaîtrait «son goût pour ce genre de symétrie qui aime les combinaisons compliquées, qui se plaît à diviser et subdiviser indéfiniment, toujours d'après le même ordre, précisément comme dans les églises gothiques $»^{21}$. Le style gothique reposerait donc, à en croire Schopenhauer, sur une logique d'exposition discursive analytique. C'est la même idée que l'on retrouvera développée par Erwin Panofsky en 1951 ; mais avant Panofsky, plusieurs historiens allemands, dans la première moitié $\mathrm{du} \mathrm{XX}^{\mathrm{e}}$ siècle, auront d'abord accrédité cette lecture et répandu l'idée d'une vision totalisante, unifiant, dans un plan d'ensemble, jusqu'au moindre détail, et dont la lumière serait le liant. L'esthétique gothique aurait alors une vocation gnoséologique : celle de traduire et de manifester esthétiquement l'accord structurel entre la raison discursive et l'expression holistique de la transcendance.

18. Goethe, Von der Deutsche Baukunst (1823), in Sämtliche Werke, Munich, Carl Hanser Verlag, 1993, vol. 13.2, « Die Jahre 1820-1826», p. 164.

19. Hegel, Cours d'esthétique, trad. J.-P. Lefebvre et V. von Schenk, Paris, Aubier, 1996, p. 331. Pour une analyse esthétique de la définition hégélienne de la cathédrale et du style gothique, qui est hors de notre propos ici, nous renvoyons à notre thèse « Le concept philosophique du gothique », soutenue à l'Université Paris I, p. 343-354.

20. Traduit et cité par W. Schlink, «Existait-il un programme d'ensemble pour les cathédrales au Moyen-Âge? », trad. A. Virey-Wallon, in Le monde des cathédrales, sous la direction de R. Recht, Actes du colloque organisé par le Musée du Louvre, Paris, La Documentation Française, 2003, p. 16.

21. Schopenhauer, Le Monde comme volonté et comme représentation, trad. A. Burdeau, Paris, P.U.F./Quadrige, 2004. 
Ces analyses font l'objet de plus en plus de critiques. Wilhelm Schlink, dans un cycle de conférence en 2000 qui reprend les avancées de la recherche sur ce point à la fin du $\mathrm{XX}^{\mathrm{e}}$ siècle, se propose de « mettre en doute la "belle" unité de la cathédrale gothique $»^{22}$. Il pointe certains historiens allemands et leur enthousiasme holistique pour le moins suspect. Hans Jantzen d'abord: "La lumière pourpre de la cathédrale de Chartres contribue à l'impression de ravissement suscitée par l'espace intérieur $»^{23}$. Hans Sedlmayr (dont Wilhelm Schlink soulignera, à la suite de Roland Recht, que son engagement aux côtés des nazis n'était sans doute pas étranger à ses analyses esthétiques) ensuite: "Que l'ardente lueur des verrières de la cathédrale évoque des parois de pierres précieuses est une évidence [...]. La myriade de leurs formes individuelles font de la cathédrale le reflet du monde céleste $»^{24}$.

L'idée développée par ces historiens (et d'autres) est la suivante : si la cathédrale gothique, dans sa structure même, correspond bien à un programme d'ensemble, dans le souci de manifestation d'une totalité harmonieuse, alors la lumière est ce qui permet d'unifier, dans le volume de l'édifice, la multiplicité de détails formels et la variété de l'iconographie, mêlant les scènes les plus diverses de l'Ancien ou du Nouveau Testament. Pour soutenir que les bâtisseurs avaient en tête de figurer une unité surnaturelle, il faut que le volume soit baigné de lumière céleste (car pour Sedlmayr «ici nous sommes au Ciel et c'est moins l'instruction qui nous attend que l'illumination $»^{25}$ ). Or, analyse Wilhelm Schlink, aucun programme d'ensemble ne saurait être reconnu de manière indiscutable. Peter Kurmann, dans le même cycle de conférences, reprend cette idée de figuration céleste ou surnaturelle et, s'il accorde que « du point de vue théologique, tout édifice religieux consacré est une représentation de la Jérusalem céleste ${ }^{26}$, il considère qu'il faut néanmoins abandonner «définitivement l'idée que la cathédrale gothique a exprimé l'image de la Jérusalem céleste par sa structure et par ses formes architecturales ${ }^{27}$. Il partage avec Roland Recht et Wilhelm Schlink l'idée que Sedlmayr et les autres, faisant de la cathédrale un monument «surnaturel», se paient $d^{\prime}$ ' interprétations entièrement gratuites dont on chercherait en vain le fondement historique dans les sources liturgiques ou littéraires du MoyenÂge ${ }^{28}$.

22. W. Schlink, «Existait-il un programme d'ensemble pour les cathédrales du MoyenÂge? ", Le monde des cathédrales, op. cit., p. 15.

23. Ibid., p. 17.

24. Ibid.

25. Ibid.

26. P. Kurman, «La cathédrale gothique est-elle l'image de la Jérusalem céleste ? », in Le monde des cathédrales, op. cit., p. 45.

27. Ibid., p. 50.

28. Ibid. 
Ni «programme d'ensemble », ni image fidèle de la « Jérusalem céleste »: on ne voit plus alors très bien la justification de la volonté totalisatrice et unificatrice qui aurait présidé à l'élaboration conceptuelle des cathédrales gothiques. Elle serait donc bien gothique au sens premier du terme : sans unité préalable, elle serait composée de parties indépendantes les unes des autres, assemblage d'une multiplicité sans ambition de constituer une totalité harmonieuse.

Paul Frankl, recherchant la meilleure manière de définir «l'essence du gothique », parviendra en 1960 à la conclusion suivante : «L'essence de sa forme comme du sens du gothique, c'est le parcellaire ; ce qui veut dire que chaque partie est le fragment d'un tout qui ne saurait être qu'un fragment d'infinité ${ }^{29}$. Le mot «parcellaire», partiality en anglais, est particulièrement intéressant puisqu'il signifie à la fois une subjectivité exclusive et un souci du particulier au détriment du général. Il ne faut pas le traduire en français par "partialité », qui signifie la prise en compte d'une partie au lieu d'un ensemble, mais par " parcellaire », que nous entendons comme une partie d'un ensemble qui ne peut pas être totalisé. Le parcellaire s'illustre exemplairement dans les très nombreuses cathédrales où les deux clochers qui dominent la façade ne sont pas similaires (où parfois le second n'est même pas construit - comme à Strasbourg...). Certes, cela s'explique historiquement par la longue durée des travaux, ou par les destructions (incendies ou catastrophes) imposant des reconstructions ; mais nous voyons bien, au-delà de ces raisons contingentes, que les maîtres d'œuvre ne se sentaient nullement obligés de construire (ou reconstruire) les tours à l'identique. Cela aurait été intolérable dans une œuvre classique ou l'harmonie est le maître-mot, puisque c'est elle qui produit l'idée de totalité, but ultime de l'œuvre. En revanche, dans le style gothique, chaque tour est une œuvre en elle-même et se passe parfaitement de l'appréciation générale d'un ensemble qui n'est pas valorisé en tant que totalité unifiée. C'est là, exemplairement, l'esprit du parcellaire qui est à l'œuvre. Cet esprit, qui selon Frankl constitue «l'essence du gothique », s'explique donc moins par un souci du détail, qu'on trouve aussi bien dans d'autres styles ${ }^{30}$, qu'à un processus d'effacement des limites totalisatrices de l'édifice causé par la surenchère de détails. Ce que Frankl reprécise quelques lignes après :

L'essence du gothique peut donc être décrite comme le principe du parcellaire appliqué à l'architecture des églises romanes, qui existait déjà, à

29. P. Frank1, The Gothic. Literary sources and Interpretations through Eight Centuries, Princeton University Press, 1960, p. 830. Nous traduisons : Both the meaning and the form of this essence are partiality; that means that each part is a fragment of a whole which itself tends to be only a fragment of infinity.

30. Partiality is the essence of many another style and consequently the essence of Gothic is not exhausted by this one word. It is characteristic of the essence of Gothic that this partiality came to dominate church building in its earliest stage of Romanesque (ibid.). Nous traduisons : "Autrement dit, le gothique ne se réduit pas à une esthétique du parcellaire, mais à une volonté de s'imposer contre l'idée classique d'une limitation totalisante de l'édifice ». 
partir de 1093 environ. Cette essence était dotée de la puissance créatrice de produire des nouvelles formes en harmonie avec elle-même, et peu à peu s'est libérée de tout ce qui était roman et « total»

Deux objections doivent maintenant être considérées. La première porte bien sûr sur le rôle des vitraux. N'ont-ils pas pour vocation, comme le pensaient Jantzen ou Sedlmayr, d'illuminer «l'espace intérieur» et ce faisant d'unifier les différentes dimensions de l'édifice au sein d'une totalité diaphane et harmonieuse ? Certes, toutes les recherches sur l'art des vitraux mettent constamment en avant l'art de la lumière et l'ingéniosité des coloristes : «Or, combien de théories - celles sur la diaphanéité en particulier -, combien d'interprétations formalistes tendant à définir les qualités spécifiques de l'espace gothique se trouvent ruinées lorsque l'œil cherche à lire l'intérieur de l'édifice gothique non plus comme un jeu de formes tridimensionnelles articulées, mais comme une construction illusionniste à laquelle conduisent des effets colorés, pouvant contrarier l'architecture ${ }^{32}$, remarque Roland Recht.

Ce dernier invite à se séparer pour de bon d'un présupposé qui a la vie dure : les vitraux ne sont pas des fenêtres qui ouvriraient la cathédrale sur le monde extérieur. S'ils utilisent avec ingéniosité la lumière naturelle, ce n'est pas pour éclairer l'intérieur mais pour briller, et ainsi mettre en valeur les scènes qu'ils représentent. Éclairer et briller sont deux choses bien différentes. L'historien des couleurs Michel Pastoureau a bien montré comment, depuis la basse antiquité au moins, on pouvait définir chaque couleur non pas en fonction de sa valeur chromatique mais en fonction de sa brillance :

Pour nommer la couleur, le paramètre de luminosité est plus important que celui de la coloration. Le lexique cherche d'abord à dire si la couleur est mate ou brillante, claire ou sombre, dense ou diluée, ensuite seulement si elle s'inscrit dans la gamme des blancs, des noirs, des rouges, des verts, des jaunes ou des bleus. [...] Le rapport à la lumière prime tout le reste ${ }^{33}$.

Cela s'applique à l'art des vitraux : « Pour l'homme du Moyen-Âge, la lumière était une des formes de manifestation de Dieu, si bien qu'il voyait dans les vitraux translucides des représentations de la parole du Seigneur. Les théologiens leur attribuait la vertu d'éclairer l'humanité et de l'écarter du mal», souligne Brigitte Kurman-Schwarz ${ }^{34}$. Roland Recht rappelle que

31. Ibid., p. 831. Nous traduisons: The essence of Gothic can thus also be described as the principle of partiality applied to the historically existing Romanesque church architecture from about 1093 on, and this essence was endowed with the creative power to produce new forms that were in harmony with itself, that gradually freed it from everything Romanesque and "total".

32. R. Recht, Le Croire et le voir, Paris, Gallimard, 1999, p. 229.

33. M. Pastoureau, Noir, histoire d'une couleur, Paris, Seuil, 2008, p. 28.

34. B. Kurman-Schwarz, "Le Vitrail gothique », L'Art Gothique, sous la direction de Rolf Toman, Cologne, Könemann, 1998, p. 469. 
« avant de constituer une apologie de la lumière, le vitrail est une image, et en tant que tel, doit être lu comme un récit $»^{35}$. Ces remarques doivent nous interroger: comment cela aurait-il été possible si le rôle du vitrail était d'éclairer? Le vitrail aurait été éblouissant et par conséquent illisible. Autant vouloir regarder le soleil en face. Ce qu'on appelle la «théologie de la lumière » ne saurait donc être compris en un sens moderne qui consisterait à dissiper l'obscurité au profit d'un «tout-lumineux». C'est bien plutôt l'inverse : l'obscurité envahissant tout le volume de la cathédrale permet de laisser contraster efficacement les brillances des vitraux. Celles-ci ressortent d'autant plus que la pénombre règne à l'intérieur. Ils servent de modèles spirituels à la conversion du pèlerin qui se retrouve perdu dans l'ombre de la cathédrale comme l'homme pécheur dans le monde.

Malgré cela, une volonté moderne, depuis deux siècles au moins, est à l'œuvre, pour dissiper à tout prix cette obscurité. Elle passe en premier lieu par la pose de vitres blanches translucides en remplacement des vitraux colorés. Victor Hugo, déjà, dénonçait cette tentative de récupération grossière par la pensée classique de la cathédrale gothique :

Et qui a mis de froides vitres blanches à la place de ces vitraux « hauts en couleur » qui faisaient hésiter l'œil émerveillé de nos pères entre la rose du grand portail et les ogives de l'abside ? Et que dirait un sous-chantre du seizième siècle, en voyant le beau badigeonnage jaune dont nos vandales archevêques ont barbouillé leur cathédrale ? [...] Il croirait que le lieu saint est devenu infâme, et s'enfuirait ${ }^{36}$.

La restauration, partout en Europe, des cathédrales partiellement détruites au cours du $\mathrm{XX}^{\mathrm{e}}$ siècle, a souvent été le prétexte tout trouvé pour faire poser ces vitres blanches. Aujourd'hui, à grand renfort de lumières artificielles, l'ombre qui les constituait se voit dans certains cas définitivement dissipée par une clarté de mauvais aloi, mais qui - c'est là le point important - semble plaire et réconforter l'esprit moderne. Si le désir de faire de la cathédrale un monument lumineux est si important aujourd'hui, c'est peut-être parce que le rôle esthétique de la lumière se double d'une valeur noétique indéniable: la pensée n'est-elle pas constamment comparée, voire identifiée à une instance éclairante? Ne voitelle pas ses représentations; ne fait-elle pas la lumière sur quelque objet en résolvant les problèmes qui lui sont liés ; n'est-elle pas lucide quand elle se représente le monde tel qu'il est objectivement (ou du moins le croit-elle) ? Le règne de la lumière, c'est la traduction esthétique du règne de la pensée. Et comment la cathédrale, édifice si ingénieux et si ambitieux, pourrait-elle être autre chose qu'un chef-d'œuvre de la pensée humaine travaillant, au fond, pour sa propre gloire?

35. R. Recht, Le Croire et le voir, op. cit., p. 240.

36. V. Hugo, Notre-Dame de Paris, Paris, Folio Gallimard, 1984, p. 190. 
Il faut à ce stade considérer le cas fondateur de la cathédrale de SaintDenis, et de la théologie de la lumière que l'abbé Suger a voulu y incarner :

L'œuvre resplendit d'une noble lumière. Que son éclat

Illumine les esprits afin que, guidés par de vraies clartés,

Ils parviennent à la vraie lumière, là où le Christ est la vraie porte ${ }^{37}$.

Roland Recht nous avertit du piège qu'il y aurait à se contenter de ces quelques vers : "Depuis Panofsky, on a lu ces vers dans la perspective des doctrines néoplatoniciennes, à juste titre, mais, nous semble-t-il, c'est insuffisant $»^{38}$. En outre, considérer Saint-Denis comme le seul ou même le premier foyer du gothique, donnant au style entier une direction esthétique univoque, reviendrait à commettre une grossière erreur. Entre autres, l'intérêt de la cathédrale de Sens, dont le chantier commence en 1140 « dépasse peut-être celui de Saint-Denis » ${ }^{39}$. Voici comment Roland Recht y analyse le rôle de la lumière :

Le traitement de la lumière est ici beaucoup plus nuancé que dans le déambulatoire de Suger, où la communication entre les chapelles rayonnantes, ajoutée à la finesse des supports, conférait une fonction déterminante aux verrières. À Sens, plusieurs données s'opposent à une telle glorification de la lumière : l'obscurité qui règne dans les fausses tribunes et l'existence de voûtes sexpartites dont les voûtants exercent, en quelque sorte, la fonction d'œillères de part et d'autre des baies ${ }^{40}$.

Théologie de la lumière ne veut pas dire omniprésence de la lumière, mais jeu de lumières et d'ombres de manière à établir une dramaturgie, puisqu'il s'agit de rendre visible le mystère de la foi. Le mystère et l'inquiétude suscités par l'obscurité contrastent avec la lumière et établissent un espace « cinétique » ${ }^{41}$ qui porte toute l'attention du fidèle vers le chœur, centre lumineux qui se détache de l'abside obscure (comme à Chartes, Reims, Paris ou Amiens). Le fidèle est «porté » de l'ombre à la lumière, à travers un chemin de conversion. «En cela, dit Roland Recht, nous sommes plus proches de la mystique bernardienne que de celle de Suger [...]. Ainsi la vérité, au dire de Saint Bernard, vient de l'interaction contrastée de l'ombre et de la lumière ${ }^{42}$.

Pourtant - et c'est là la deuxième objection qu'il nous faut considérer les travaux d'Erwin Panofsky, qui firent date dans l'histoire des interprétations de l'art gothique, ont largement contribué à faire de Suger

37. Cité et traduit par R. Recht, Le Croire et le voir, op. cit., p. 150.

38. Ibid., p. 150.

39. Ibid., p. 157.

40. Ibid., p. 160.

41. Ibid., p. 165.

42. Ibid., p. 160. 
et de l'esthétique néoplatonicienne de Saint-Denis, la matrice du style gothique : «Suger eût-il conscience [qu'il] inaugurait cette grande synthèse sélective de tous les styles régionaux qui, dans cette Île-de-France jusque-là relativement stérile, devait donner ce que nous appelons le style gothique ? [...] A-t-il su ou pressenti que son enthousiasme instinctif pour la métaphysique de la lumière du Pseudo-Aréopagite et de Jean-Scot Erigène, le plaçait à l'avant-garde d'un mouvement intellectuel ?» ${ }^{43}$.

Dans un texte décisif sur le sujet, Architecture gothique et pensée scolastique, traduit en français par Pierre Bourdieu, Erwin Panofsky part de la coïncidence troublante (qui n'en est pas une, selon lui) de la naissance de la scolastique et de l'architecture gothique, pour tenter de montrer qu'elles partagent le même souci d'accorder la raison et la foi. Pour lui, au tournant du XII ${ }^{\mathrm{e}}$ siècle, les architectes travaillant en Île-de-France ont en commun avec les scolastiques un souci de transparence logique ${ }^{44}$ : les uns rendent manifeste (manifestatio) l'architecture logique de leurs raisonnements expurgés de toute rhétorique tandis que les autres construisent en dehors de l'édifice les arcs-boutants qui le soutiennent; tous sont alors censés viser une fin identique: l'expression de la totalité et d'une concordance (concordantia) entre la pensée immanente et le réel transcendant. Panofsky se rattache ainsi explicitement à la tradition interprétative holistique ${ }^{45}$, avec cette originalité qui fait de la construction gothique le pendant architectural de la construction métaphysique classique dionysienne et néoplatonicienne :

Comme la Summa scolastique, la cathédrale classique vise, avant tout, à la totalité et tend par conséquent à s'approcher autant que possible d'une solution parfaite et ultime; on peut donc, à propos du gothique classique, parler d'un plan ou d'un système avec plus d'assurance qu'à propos de n'importe quelle autre période ${ }^{46}$.

Bien sûr, le terme de "classique " ne renvoie pas ici à une période artistique historiquement délimitée. Il a néanmoins un double sens. D'une part, Panofsky distingue un style classique qui «exige un maximum d'harmonie (un style impeccable dans l'écriture, une proportion impeccable) $\gg{ }^{47}$ d'un style gothique qui, tout comme la scolastique, «réclame un maximum d'explicitation ${ }^{48}$. D'autre part, ces deux styles

43. E. Panofsky, «L'abbé Suger de Saint-Denis», in Architecture gothique et pensée scolastique, trad. P. Bourdieu, Paris, Editions de Minuit, 1967, p. 63.

44. Ibid., p. 102 : «De même que la scolastique classique est dominée par le principe de manifestatio, de même l'architecture gothique classique est dominée, comme l'observe déjà Suger, par ce que l'on peut appeler le "principe de transparence" ».

45. Erwin Panofsky l'admet dans la dernière page du texte : " Peut-être [le lecteur] objecterat-il que l'évolution esquissée ici n'est qu'une actualisation du schème hégélien ». Ibid., p. 130 .

46. Ibid., p. 103. Nous soulignons.

47. Ibid., p. 112.

48. Ibid., p. 113. 
atteignent leur «classicité » quand ils répondent au mieux à l'idéal fixé, ou, comme l'explique Pierre Bourdieu, à «leurs propres normes de perfection ${ }^{49}$ : proportion dans un cas, clarification logique dans l'autre. Ainsi, le style gothique atteint un niveau classique suivant une définition générale donnée dans L'œuvre d'art et ses significations, c'est-à-dire une règle esthétique d'origine grecque et qui «a identifié le principe de beauté à l'accord des parties entre elles et avec le tout ${ }^{50}$. Or cette règle de classicité est transhistorique et s'applique tant au style classique (grec et romain) ou néoclassique qu'au style gothique. Quant à ce dernier, sa manière originale d'être classique réside dans la manifestation des articulations et des enchaînements de formes variées, de manière à susciter un sentiment de parachèvement de la pensée.

On comprend mieux, dès lors, pourquoi toute mention du parcellaire et de l'obscur a disparu de l'analyse de Panofsky: le moindre détail est illuminé pour mieux renvoyer à la totalité " parfaite et ultime» de l'édifice. Panofsky y voit une mécanique de «l'illumination anagogique ${ }^{51}$ permettant à la pensée de s'élever de la cathédrale matérielle à la cathédrale spirituelle, puisque «notre esprit, dit le Pseudo-Aréopagite $[\ldots]$, peut s'élever à ce qui n'est pas matériel sous la conduite de ce qui l'est $\gg{ }^{52}$.

C'est tout l'univers matériel qui devient une grande « lumière », composée d'une infinité de petites lumières qui sont comme autant de lanternes [...]; chaque chose perceptible, qu'elle soit l'œuvre de l'homme ou naturelle, devient un symbole de ce qui n'est pas perceptible, une marche pour l'ascension vers le ciel ; l'esprit humain lorsqu'il s'abandonne à «l'harmonie et au rayonnement» (bene compactio et claritas) qui sont le critère de la beauté terrestre, se sent lui-même " guidé » vers la cause transcendante de cette « harmonie » et de ce «rayonnement $»$, qui est Dieu ${ }^{53}$.

Autrement dit, il s'agirait de s'élever du fini à l'infini « en conférant une expression visuelle au concept d'infini ${ }^{54}$ : Panofsky, s'il se rattache à cette vision de la cathédrale comme illumination céleste, est celui qui lui donne, grâce au concept d'infini l'assise philosophique la plus solide. Certes, Panofsky, après Hegel, Schlegel et d'autres, identifie avec précision l'effet d'infini, les moyens esthétiques mis en œuvre pour l'exprimer (notamment l'effet de perspective) et les nouvelles inquiétudes philosophiques qui le sous-tendent. Mais c'est sur la signification et la nature de cet infini gothique qu'il reste fidèle à la tradition holistique, en en faisant un infini transcendant, continu au fini, éclairant, ou plutôt illuminant, la pensée.

49. P. Bourdieu, « Postface », in Architecture gothique et pensée scolastique, ibid., p. 163.

50. E. Panofsky, L'œuvre d'art et ses significations, trad. Marthe et Bernard Teyssèdre, Paris, Gallimard, 1969 (1955), p. 152.

51. Ibid., p. 43.

52. Ibid., p. 39.

53. E. Panofsky, Architecture gothique et pensée scolastique, op. cit., p. 40.

54. Ibid., p. 79. 
Mais Dieu, à l'époque, n'est pas à proprement parler infini. Il est incompréhensible et immense $e^{55}$, c'est-à-dire incommensurable du fait de sa grandeur excédant la mesure de la pensée, ainsi que l'a formulé Anselme de Cantorbéry ${ }^{56}$. L'infinité garde encore beaucoup de la valeur péjorative qu'elle a en grec : apeira et aoristia, l'illimité et l'indéterminé, sont les déterminations négatives du chaos et de ce qui ne ressort pas de l'ordre du cosmos. L'infinité de la cathédrale n'est pas l'immensité de Dieu : c'est une infinité sombre, négative, qui renvoie plutôt à l'égarement dans laquelle la pensée se perd. Autrement dit, sa hauteur et sa verticalité n'ont pas pour but d'élever la pensée, mais de la laisser interdite afin qu'il ne reste plus au pèlerin écrasé sous son poids qu'à s'abandonner à la transcendance divine.

Tout se passe alors comme si l'élan ascendant imprimé par la hauteur permettait à l'âme de se dépouiller de son immanence, dans un mouvement d'écrasement, comme que le suggère Bruno Klein à propos de la cathédrale d'Amiens, commencée en 1220 par Robert de Luzarches :

Celui qui pénètre dans l'église par le portail occidental ne rencontre rien à hauteur du regard : les portants extrêmement minces relèguent dans des zones inaccessibles les parois de la nef centrale [...]. La zone du portail n'offre à hauteur de regard que des petits reliefs, tandis que les scènes de l'histoire sainte qui se trouvent au-dessus se dérobent encore davantage à nous que celles des édifices précédents. Ils obligent les fidèles levant la tête à les voir par en-dessous, une attitude qu'ils devront également prendre à l'intérieur pour admirer l'architecture ${ }^{57}$.

Mais au-delà de la seule hauteur écrasante de la cathédrale d'Amiens (après Chartres, Soissons et Bourges, et juste avant celle de Beauvais commencée en 1226), c'est une ambition primordiale du style gothique qui se trouve dégagée et, à travers elle, sa dimension philosophique et noétique. Car cette hauteur écrasante des vitraux et des triforiums n'a pas d'autre effet, comme nous venons de le voir, que celui de perdre le regard, et, avec lui, la pensée. Ainsi la dramaturgie de la cathédrale, ou son «espace cinétique ", pour reprendre l'expression de Roland Recht, est celui d'une démarche de conversion, d'une élévation de l'âme vers Dieu rendue possible par ce trouble de la pensée et dont l'extase mystique est la forme la plus radicale. On peut trouver une transcription philosophique assez révélatrice de cette élévation de l'âme accompagnée d'une stupeur de la pensée, dans celle décrite par Rousseau au sein de la « cathédrale » de la nature :

55. Antoine Côté, dans L'infinité divine dans la théologie médiévale 1220-1255, (Paris, Vrin, 2002), montre que c'est d'abord la notion d'immensité qui permet d'affirmer l'incompréhensibilité positive de Dieu, et non celle d'infini qui équivaut encore à une imperfection.

56. «Seigneur, Tu n'es pas seulement tel que plus grand ne peut être pensé, mais Tu es quelque chose de plus grand qu'il ne se puisse penser », Anselme de Cantorbéry, Proslogion, trad. M. Corbin, Paris, Cerf, 1986, ch. XV, p. 267.

57. Bruno Klein, «Naissance et formation de l'architecture gothique en France et dans les pays limitrophes ", L'Art Gothique, op. cit., p. 64. 
Alors, l'esprit perdu dans cette immensité, je ne pensais pas, je ne raisonnais pas, je ne philosophais pas ; je me sentais avec une sorte de volupté accablé du poids de cet univers, je me livrais avec ravissement à la confusion de ces grandes idées, $\mathrm{j}$ 'aimais à me perdre en imagination dans l'espace, mon cœur resserré dans les bornes des êtres s'y trouvait trop à l'étroit, j'étouffais dans l'univers, $\mathrm{j}$ 'aurais voulu m'élancer dans l'infini. Je crois que si j'eusse dévoilé tous les mystères de la nature, je me serais senti dans une situation moins délicieuse que cette étourdissante extase à laquelle mon esprit se livrait sans retenue, et qui, dans l'agitation de mes transports, me faisait écrier quelquefois : « Ô grand Être ! Ô grand Être !» sans pouvoir dire ni penser rien de plus ${ }^{58}$.

Le délice avec lequel Rousseau laisse stupide (c'est-à-dire frappée de stupeur) la pensée, dans le but de mieux se livrer au ravissement esthétique d'un environnement, n'est pas sans rappeler la fascination inquiète produite par l'esthétique gothique du parcellaire et de l'obscur. La pensée " étouffe » alors même que les affects esthétiques donnent l'impression de «s'élancer dans l'infini ». La pression de l'infini frappe la pensée en même temps qu'elle excite la sensibilité. Dans les deux cas, la pensée pâtit de la pression d'une réalité incommensurable: celle, délicieuse, de la nature chez Rousseau, et celle, inquiétante, produite par l'espace dramatisé de la cathédrale gothique. Mais à chaque fois, l'effet recherché est celui de ravissement. L'expression esthétique de cette pression de l'infini est à la base de l'« intention gothique », selon l'expression de Wilhelm Worringer, qui préside à l'érection de la cathédrale.

Ce n'est pas par joie de construire que le gothique bâtit ses cathédrales jusqu'à l'infini, il veut que l'aspect de ce mouvement vertical, qui dépasse de loin toute échelle humaine, provoque en lui le vertige de la sensation où seul il peut endormir ses démons intérieurs et trouver la béatitude. La beauté du fini suffisait à l'homme classique pour son élévation intérieure, mais le gothique déchiré par le dualisme [...] ne pouvait sentir que dans l'infini le frisson de l'éternités.

Worringer, à partir de la verticalité à double sens de l'infini, pose les termes de l'opposition entre gothique et classique dans sa radicalité. Dans l'esthétique gothique, la pression de l'infini, en cohérence avec l'esthétique de l'obscur et du parcellaire identifiée par les historiens, est bien éloignée de la recherche classique d'un environnement proportionné à la mesure de l'homme. Elle laisse au contraire entrevoir une réalité disproportionnée « qui dépasse de loin toute échelle humaine ».

S'interrogeant sur les raisons qui ont poussé les néo-classiques à condamner si vivement l'art gothique, Robson-Scott remarque que « selon

58. J.-J. Rousseau, Troisième lettre à M. de Malesherbes, in CEuvres Complètes, tome I, Paris, Gallimard Pléiade, 1959, p. 1140.

59. W. Worringer, L'Art gothique, (Formprobleme der Gotik, 1927), traduction française par

D. Decourdemanche, Paris, Gallimard, 1967 (1941), p. 158. 
l'esthétique néo-classique, les qualités constitutives de la beauté classique dans le domaine architectural sont avant tout la simplicité, la régularité, de justes proportions, de la propriété. Le style gothique est une offense à toutes ces qualités. Il doit par conséquent être condamné $\gg{ }^{60}$. La simplicité, la régularité, l'harmonie des proportions caractérisent l'ordre ici défini comme « classique » ou " néo-classique ». Mais, dans la cathédrale gothique, tout est fait pour que ces éléments soient absents : elle est une anti-unité par son obscurité, une anti-totalité par sa dimension parcellaire, une anti-perfection par sa disproportion.

Nous avons déjà dit comment ce triple refus abandonnait la pensée dans un égarement perceptif et, par suite, gnoséologique. Son environnement n'est plus alors perçu comme familier ou domestique, mais étranger, voire fantastique. Les développements littéraires et picturaux de la mode néogothique dans l'Angleterre des $\mathrm{XVIII}^{\mathrm{e}}$ et $\mathrm{XIX}^{\mathrm{e}}$ siècles montrent comment la forme gothique du $\mathrm{XIII}^{\mathrm{e}}$ siècle est particulièrement bien adaptée au gloomy et à l'expression mélancolique de distance et d'étrangeté par rapport au monde, notamment à travers sa représentation sous forme de ruine éclairée par la lune : comme si, paradoxe suprême, la cathédrale médiévale trouvait sa forme la plus accomplie dans celle de la ruine ${ }^{61}$. Le néogothique va ainsi constituer une sorte de réponse à l'interprétation classique de l'architecture gothique, en mettant en avant sa part d'obscur et de parcellaire, et l'effet d'infini qui pèse sur la pensée plus qu'il ne la supporte. On peut légitimement se demander comment une architecture qu'on voudrait entièrement dévouée au diaphane et à l'évocation céleste peut si complaisamment se prêter aux effets lugubres et fantastiques d'une esthétique dominée par l'obscurité. En France, c'est Victor Hugo qui nous oriente vers la réponse néogothique en prenant résolument le contrepied de l'interprétation holistique et en faisant de la cathédrale un monstre de noirceur. Lisons, entre cent autres, le passage où l'archidiacre Claude Frollo, persuadé que La Esmeralda est morte sur son ordre, en proie à la démence, se réfugie dans Notre-Dame :

Il trouva dans l'église une obscurité et un silence de caverne. [...] La grande croix d'argent scintillait au fond des ténèbres, saupoudrée de quelques points étincelants, comme la voie lactée de cette nuit de sépulcre. Les longues fenêtres du chœur montraient au-dessus de la draperie noire l'extrémité supérieure de leurs ogives, dont les vitraux, traversés d'un rayon de lune,

60. W. D. Robson-Scott, The literary Background of the Gothic Revival in Germany, op. cit., p. 15. Nous traduisons: The constituent qualities of classical beauty in the architectural sphere, according to the neo-classical aesthetic, were above all simplicity, regularity, just proportions, propriety. The Gothic style offended against all these. Therefore it must be condemned.

61. Parmi les très nombreux travaux sur le sujet, bornons-nous à citer l'ouvrage de référence en français sur le sujet, écrit par Maurice Lévy : Le Roman « gothique » anglais 1764-1824, Paris, Albin Michel, 1995. 
n'avaient plus que les couleurs douteuses de la nuit, une espèce de violet, de blanc et de bleu dont on ne retrouve la teinte que sur la face des morts ${ }^{62}$.

L'intention de cet extrait est manifeste : la cathédrale, envahie par une « nuit de sépulcre », devient une figuration de la tombe que Frollo creuse luimême par sa folie de vengeance et son délire de culpabilité. Mais si l'archidiacre trouve dans la cathédrale une projection si puissante de ses hallucinations morbides, c'est aussi peut-être que l'esthétique de l'édifice n'y est pas étrangère. Une telle vision de la cathédrale, qui contredit si fortement la lecture classique, n'est pas moins légitime qu'elle, quoiqu'elle lui répugne. Car celle-ci se borne à lire la cathédrale de jour, passant délibérément sous silence les heures de la nuit, niant implicitement qu'elles puissent être d'une quelconque importance dans la compréhension globale du style gothique.

C'est bien sûr tout le contraire : au cours de la nuit, la dimension de négation de l'immanence humaine au profit d'un abandon de soi dans la dynamique transcendante se trouve être puissamment renforcée. Hugo suggère que l'architecture des cathédrales gothiques, sans éclairage artificiel, renforce l'obscurité naturelle de la nuit jusqu'à lui conférer une dimension fantastique qui souligne l'égarement, voire l'affolement de la pensée qui se trouve comme dessaisie d'elle-même. La lumière de la lune, beaucoup plus pâle et moins brillante, fait luire les vitraux sans les faire briller, ce qui a pour effet de rendre plus impénétrable encore l'obscurité de la cathédrale.

La cathédrale fonctionne alors comme une sorte de miroir noétique. Si, d'un côté, elle montre la puissance logique et discursive que la pensée est capable de développer pour évoquer une transcendance, de l'autre elle en souligne la fragilité dès qu'il s'agit de se mesurer à elle. L'effet d'infini fonctionne dans les deux sens. Il est ascendant et dynamique pour porter le fidèle vers Dieu dans un effet de ravissement; il est étouffant et panique pour rappeler à la pensée l'étrangeté irréductible du réel d'un monde qui n'est pas à la mesure de l'homme, mais à la mesure de Dieu. Dans les deux cas, il constitue pour la pensée un dessaisissement. Loin d'évoquer une harmonie entre puissance noétique et transcendance divine, la cathédrale gothique montre au contraire la disproportion incommensurable de l'une à l'autre, et l'inadéquation de la raison discursive au mystère de la foi. On reste sur ce sentiment curieux que jamais la pensée n'aura mis autant de soin à se dépasser et à s'invalider elle-même. 